\title{
Repurposing tebipenem pivoxil as alternative therapy for severe gastrointestinal infections caused by extensively-drug resistant (XDR) Shigella spp.
}

\section{Elena Fernández Alvaro}

GSK Global Health, Tres Cantos, Madrid

\section{Phat Voong Vinh}

The Hospital for Tropical Diseases, Wellcome Trust Major Overseas Programme, Oxford University Clinical Research Unit, Ho Chi Minh City, Vietnam

\section{Cristina de Cozar}

GSK Global Health, Tres Cantos, Madrid

\section{Beatriz Urones}

GSK Global Health, Tres Cantos, Madrid

\section{Alvaro Cortés}

GSK Global Health, Tres Cantos, Madrid

\section{Alan Price}

GSK Global Health, Tres Cantos, Madrid

\section{Nhu Tran Do Hoang}

OUCRU

\section{Ha Thanh Tuyen}

Oxford University Clinical Research Unit

\section{Molly McCloskey}

Division of Allergy and Infectious Disease, Center for Emerging and Re-emerging Infectious Diseases, University of Washington School of Medicine

\section{Shareef Shaheen}

Division of Allergy and Infectious Disease, Center for Emerging and Re-emerging Infectious Diseases, University of Washington School of Medicine

\section{Denise Dayao}

Department of Infectious Disease and Global Health, Tufts University Cummings School of Veterinary Medicine, North Grafton, Massachusetts

\section{Jaime de Mercado}

GSK Global Health, Tres Cantos, Madrid

\section{Adolfo García-Perez}

GSK Global Health, Tres Cantos, Madrid 


\section{Benson Singa}

Kenya Medical Research Institute, Nairobi

\section{Patricia Pavlinac}

Department of Global Health, University of Washington, Seattle, Washington

\section{Judd Walson}

Division of Allergy and Infectious Disease, Center for Emerging and Re-emerging Infectious Diseases, University of Washington School of Medicine, Seattle, Washington

\section{Maria Santos Martínez-Martínez}

GSK Global Health, Tres Cantos, Madrid

\section{Samuel Arnold}

Division of Allergy and Infectious Disease, Center for Emerging and Re-emerging Infectious Diseases, University of Washington School of Medicine, Seattle, Washington

\section{Saul Tzipori}

Tufts University

\section{Lluis Ballel}

GlaxoSmithKline

\section{Stephen Baker ( $\nabla$ sgb47@medschl.cam.ac.uk)}

University of Cambridge https://orcid.org/0000-0003-1308-5755

\section{Article}

Keywords: Shigella spp., drug discovery, multi-drug resistance, dysentery, diarrhoea, carbapenems

Posted Date: March 24th, 2021

DOl: https://doi.org/10.21203/rs.3.rs-310608/v1

License: (c) (1) This work is licensed under a Creative Commons Attribution 4.0 International License. Read Full License 


\section{Abstract}

Diarrhoea remains one of the leading causes of childhood mortality globally. Recent epidemiological studies conducted in low-middle income countries (LMICs) identified Shigella spp. as the first and second most predominant agent of dysentery and moderate diarrhoea, respectively. Antimicrobial therapy is often necessary for Shigella infections; however, we are reaching a crisis point with efficacious antimicrobials. The rapid emergence of resistance against existing antimicrobials in Shigella spp. poses a serious global health problem. Here, aiming to identify alternative antimicrobial chemicals with activity against multi-drug resistant (MDR) Shigella, we initiated a collaborative academia-industry drug discovery project, applying high throughput phenotypic screening across broad chemical diversity. We identified several suitable compounds with antibacterial activity against Shigella. These compounds included the oral carbapenem tebipenem, which was found to be highly potent against broadly susceptible Shigella and contemporary MDR variants. Additional in vitro screening demonstrated that tebipenem had activity against a wide range of other non-Shigella enteric bacteria. Cognisant of the risk for the development of resistance against monotherapy, we identified synergistic behaviour of two different drug combinations incorporating tebipenem. The orally bioavailable prodrug (tebipenem pivoxil) effectively cleared the gut of infecting organisms when administered in physiological doses to Shigella-infected mice and gnotobiotic piglets. Our data highlight the utility of broad compound screening for tackling the emerging antimicrobial resistance crisis and shows that tebipenem pivoxil (licenced for paediatric respiratory tract infections in Japan) could be repurposed as an effective treatment for severe diarrhoea caused by MDR Shigella and other enteric pathogens in LMICs.

\section{Introduction}

Humanity is currently in the midst of a global antimicrobial resistance (AMR) crisis ${ }^{1}$. Many of the antimicrobials that we have historically used to treat and/or control infections caused by common bacterial pathogens have lost or are rapidly losing their efficacy. This situation has become worryingly common for a host of diseases and pathogens, but arguably the issue is epitomised by enteric (diarrhoeal) infections that arise in low and middle-income countries (LMICs). Annually, diarrhoea accounts for approximately 1.3 million deaths globally, with the preponderance of these occurring in young children residing in LMICs ${ }^{2}$. Due to a lack of other interventions, antimicrobials are a key tool in limiting the impact of these infections, but as AMR increases these infections are likely to have an even greater impact on the most vulnerable individuals.

Diarrhoeal disease is notoriously complex to disaggregate as a wide range of pathogens can trigger the syndrome; but of all the potential aetiological agents, bacteria belonging to genus Shigella pose the greatest AMR threat. Recent estimates suggest that Shigella are associated with $\sim 125$ million diarrhoeal episodes and 200,000 deaths annually ${ }^{3}$. Additionally, Shigella (and enterotoxigenic Escherichia coli (ETEC)) were found to be the most predominant bacterial pathogens in the stools of paediatric diarrhoeal patients in South Asia and sub-Saharan Africa ${ }^{4}$. This study also found that Shigella were the most 
prevalent pathogen in diarrhoeal children aged 2 to 5 years; a reanalysis of stool samples from this study using detailed molecular approaches suggested that burden of Shigella may actual be double that of previous estimates ${ }^{5}$.

Clinical evidence strongly supports the use of antimicrobials to treat severe Shigella associated diarrhoea 6 . Appropriate antimicrobials reduce the duration of fever and diarrhoea associated with Shigella by 1-2 days, which in turn treatment limits the severity of symptoms and the risk of further complications. Additionally, the shedding of the pathogen in the stools is prevented by antimicrobials, which then reduces onward transmission. As there is currently no licenced vaccine for Shigella, antimicrobials are one of the only reliable mechanisms available for disease control. However, Shigella are highly adept at acquiring multi-drug resistance (MDR) plasmids from other member of the Enterobacteriaceae and resistance mutations are commonly associated with "successful" lineages. AMR reduces treatment efficacy and limits treatment options. Consequently, there has been an "arms race" between the pathogen and antimicrobials, with the WHO ultimately recommending ciprofloxacin, azithromycin, pivmecillinam, and ceftriaxone as the empirical drug choices for Shigella infections. Inevitably, resistance to these antimicrobials has emerged and increasingly resistant organisms, such as extensively-drug resistant (XDR) Shigella sonnei in Vietnam ${ }^{7}$, are spreading across $\mathrm{LMICs}^{8}$, in travellers returning from LMICs, and in men-who-have-sex-with-men (MSM) ${ }^{9}$. Accordingly, the US-CDC has declared AMR Shigella a serious global health threat requiring new interventions and the WHO has placed them on the global priority list of antibiotic-resistant bacteria to guide research, discovery, and development of new antibiotics ${ }^{10}$.

After the genomics revolution of the late 1990s, the research community embarked on a series of targetbased drug discovery programmes to identify novel antimicrobial compounds. This strategy, because of lack of translation from target-activity to whole-cell activity, produced largely disappointing results ${ }^{11}$. Conversely, phenotypic antimicrobial screens have proven to yield a higher hit rate than target-based approaches with several successful examples emerging in infectious diseases ${ }^{12,13}$. Aiming to identify novel compounds with activity against MDR Shigella, we established an academic-industrial collaboration ${ }^{14}$, creating a platform to perform in vitro phenotypic screening of $>1.5$ million compounds against antimicrobial susceptible and MDR Shigella. We interrogated a collecting of compounds spanning broad chemical diversity, comprised of a combination of naive chemistry, compounds originating from previous antibacterial drug discovery programs, and currently marketed compounds. Using this approach, we identified several novel options, including an oral carbapenem, that may be accelerated into further testing to treat infections caused by MDR Shigella and other gastrointestinal pathogens.

\section{Results}

\section{High throughput screening of compound collections}

We conducted an antibacterial screening campaign, incorporating both GSK proprietary and nonproprietary compounds, to identify new chemical entities (NCE), repurposing and repositioning 
opportunities that could be targeted towards treating MDR Shigella infections. The chemical diversity included the GSK screening collection ( 1.7 million compounds of unbiased chemical diversity) and 4,000 compounds with known antibacterial pedigree from the GSK antibacterial programs (chemical diversity selected by $\mathrm{MIC}<1 \mu \mathrm{M}$ against $E$. coli) and recognised, commercially available antimicrobials. A flowchart of the screening procedure is shown in Fig. 1A. The compounds were tested at a concentration of $10 \mu \mathrm{M}$ for their ability to inhibit $S$. flexneri 2457T growth in liquid medium using resazurin reduction as a surrogate of bacterial viability. Compounds fulfilling the specified hit criteria, were progressed for dose response for Shigella 2457T growth inhibition. Ultimately, we identified 2,269 compounds in seven classes (bacterial topoisomerase inhibitors (BTIs), inhibitors of UDP-3-O-( $R$-3-hydroxymyristoyl)- $N$ acetylglucosamine deacetylase ( $L p x C s)$, microcin PDI, Met-tRNA inhibitors, $\beta$-lactams, fluoroquinolones, and pleuromutilins) that inhibited Shigella 2457T growth with IC50 $\leq 1 \mu \mathrm{M}$ (Fig. 1).

\section{Two classes of antibacterial compounds exhibit activity against MDR Shigella}

The 2,269 selected active compounds identified via high throughput screening were advanced for evaluation against two clinical MDR Shigella isolates originating in Vietnam, S. flexneri EG478 and S. sonnei 02-1181; the antimicrobial susceptibility profiles of these organisms are shown in Table S1. Among all chemical classes, compounds that retained activity in the clinical isolates belonged to only three compound classes: $\beta$-lactams, BTIs, and LpxCs. The majority ( 90\%) of BTI compounds had a significant loss of potency when tested against the two clinical MDR Shigella isolates compared to the original laboratory strain (Fig. 1B). This pattern was comparable for both the LpxCs and carbapenems, with compounds generally being more active against the laboratory strain than the clinical isolates.

Given the broad availability and acceptability of carbapenems globally, efforts to determine their potential to be progressed as treatment against shigellosis were prioritized. Meropenem, tebipenem, and ertapenem exhibited the greatest bactericidal activity against the two MDR Shigella clinical isolates and were prioritized for evaluation (Fig. 1 and Table S2). Tebipenem was specifically selected for further characterization as the pivoxil prodrug has good oral bioavailability and is commercially available in Japan ${ }^{15}$. Given the route of administration and the potential for accumulation in the gastrointestinal tract, we reasoned that tebipenem may be the most appropriate identified compound that could be quickly accelerated for treating MDR/XDR Shigella infections.

\section{Tebipenem as broad-spectrum antimicrobial for treating Shigella infections}

Under direct comparison in in vitro assays, we found that tebipenem inhibited the growth of the ATCC Shigella flexneri with a potency that was comparable to currently available first line antimicrobials in broadly susceptible organisms, including both ciprofloxacin and ceftriaxone (Table 1). Given the propensity of Shigella spp. to horizontally acquire AMR genes, we assessed the activity of tebipenem against a collection of 82 contemporary clinical Shigella isolates (sonnei and flexneri) from Vietnam and 53 (a range of species) from Kenya. The compound exhibited consistent in vitro activity against all clinical isolates, with MIC values ranging from 0.04 to $0.3 \mu \mathrm{M}$ (Fig. 2A). Time kill assays of tebipenem performed in three of these Vietnamese clinical isolates (two $S$. flexneri and one $S$. sonnel) further 
confirmed potent antibacterial activity of tebipenem at 2-4x MIC over 24 hours of exposure; population rebounds started to occur after six hours exposure with 0.5-1x MICs in all three isolates (Fig. 2B).

Table 1

MIC values of different

\begin{tabular}{|ll|}
$\begin{array}{l}\text { antimicrobial for Shigella in vitro } \\
\text { extracellular growth }\end{array}$ \\
\hline Antimicrobial & $\mathbf{I C}_{90}(\mu \mathrm{g} / \mathrm{ml})$ \\
\hline Ciprofloxacin & 0.020 \\
Mecillinam & 0.058 \\
\hline Azithromycin & 7.49 \\
\hline Ceftriaxone & 0.067 \\
\hline Tebipenem & 0.010 \\
\hline
\end{tabular}

The emergence of resistance against fluoroquinolones and third generation cephalosporins has rendered them largely ineffective for the treatment Shigella in parts of South and Southeast Asia (ref). Notably, tebipenem maintained high level bactericidal activity against MDR, XDR, and fluoroquinolone resistant $S$. sonnei from Vietnam (Fig. 2A). The frequency of generating spontaneous tebipenem resistance mutations was evaluated in vitro in Shigella flexneri $2457 \mathrm{~T}$ and determined to be in a low range compared to other antimicrobials $\left(<1 \times 10^{-9}\right)$. Ceftriaxone, meropenem, and ertapenem require intramuscular or intravenous administration, thereby restricting their use for severe infections in outpatients setting. These in vitro data indicate that tebipenem administered as a prodrug may be a practical alternative for oral treatment of severe Shigella associated diarrhoea in locations with endemic MDR and fluoroquinolone resistant organisms.

As diarrhoea can be caused by multiple bacterial pathogens, we further assessed the activity of tebipenem against a range of alternative enteric bacterial pathogens using a further panel of 203 contemporary clinical isolates (Salmonella, E. coli, and Campylobacter). Tebipenem exhibited high level antibacterial activity against all non-Shigella enteric pathogens with MIC values ranging from 0.016 to 2 $\mu \mathrm{g} / \mathrm{ml}$, with a single Salmonella Enteritidis having an MIC of $4 \mu \mathrm{g} / \mathrm{ml}$ (Fig. 2A). The reported clinical MIC breakpoints for tebipenem pivoxil efficacy in the treatment of respiratory infections are $\leq 1 \mu \mathrm{g} / \mathrm{ml}$ for susceptible organisms, $\leq 2 \mu \mathrm{g} / \mathrm{ml}$ for intermediate susceptible organisms, and $\geq 4 \mu \mathrm{g} / \mathrm{ml}$ for resistant organisms ${ }^{15}$. Assuming comparable pharmacodynamics for respiratory and enteric infections, the reported MIC values indicate that tebipenem pivoxil would likely be efficacious in the treatment of all (with the exception of the single Salmonella isolate) infections caused by these bacteria.

\section{Synergistic combinations of tebipenem pivoxil}

To protect the efficacy of the drug, potentially enhance its activity, and to mitigate the risk of resistance, we explored possible synergistic combinations between tebipenem and the current WHO approved drugs 
for shigellosis; azithromycin, ciprofloxacin, ceftriaxone, and mecillinam. We additionally progressed a previous observation by combining tebipenem with a LpxC inhibitor (PF-5081090) ${ }^{16}$. Initially, these five combinations were tested against laboratory isolates of Shigella, Salmonella, and E. coli (Sf2457T, St14028, and EcDH5a, respectively) (Fig. 3A). Tebipenem exhibited partial synergy with the LpxC inhibitor, reducing the Tebipenem MIC > 10-fold in all three organisms. Similarly, tebipenem exhibited partial synergy with azithromycin against $S$. flexneri and $S$. Typhimurium, but this combination was antagonistic against $E$. coli. In combination with ciprofloxacin, ceftriaxone, and mecillinam, tebipenem exhibited indifference or partial synergy with all three organisms. This potential synergism suggests that a combination of these drugs may be formulated to protect the efficacy of tebipenem by potentially limiting the emergence of spontaneous or acquired resistance to either of the antimicrobials. Consequently, we further assessed the potential for synergism between tebipenem with the LpxC inhibitor and also tebipenem in combination with azithromycin against a panel of alternative Gram negative MDR clinical isolates. The combination of tebipenem/LpxC inhibitor exhibited indifference or partial synergy in vitro against all the tested enteric bacteria (Salmonella spp., Shigella spp., K. pneumoniae, and E. coli; Fig. 3B). A combination of tebipenem with azithromycin was partially synergistic in vitro against a range of invasive and non-invasive Salmonella isolates (Fig. 3B).

\section{Tebipenem efficacy and pharmacokinetic properties in animal models of Shigella infection}

Tebipenem is currently only approved in Japan for the treatment of respiratory tract infections. Aiming to confirm the potential of repurposing tebipenem for the treatment of enteric infections through in vivo experimentation we progressed the drug for pharmacokinetic and efficacy studies in animal models of shigellosis. The doses selected for efficacy studies were extrapolated from approved doses in children ( 4 and $6 \mathrm{mg} / \mathrm{kg} \mathrm{bid);} \mathrm{equivalent} \mathrm{doses} \mathrm{in} \mathrm{terms} \mathrm{of} \mathrm{systemic} \mathrm{exposure} \mathrm{were} \mathrm{selected.}$

The pharmacokinetics of tebipenem was characterized in mice with intravenous and oral doses of tebipenem and tebipenem pivoxil (the oral ester prodrug of tebipenem), respectively. We found that systemic concentration of active drug was equivalent after doses of $39 \mathrm{mg} / \mathrm{kg}$ of subcutaneous tebipenem and $50 \mathrm{mg} / \mathrm{kg}$ of oral tebipenem pivoxil over the course of a six-hour experiment (Fig. $4 \mathrm{C}$ and Table 2). The blood concentrations of subcutaneous tebipenem and oral gavage tebipenem pivoxil prodrug were found to be equivalent, with a comparable Cmax (71 and $62 \mu \mathrm{g} / \mathrm{mL}$, respectively) and systemic exposure ( $\mathrm{AUC}_{0-\mathrm{t}}, 80$ and $94 \mu \mathrm{g} \cdot \mathrm{h} / \mathrm{mL}$, respectively). The oral bioavailability of tebipenem when dosed as tebipenem pivoxil pro-drug was approximately 13-fold greater in mice than oral tebipenem as a free base; $39 \mathrm{mg} / \mathrm{kg}$ tebipenem free base orally had comparable bioavailability after 3 hours as $5 \mathrm{mg} / \mathrm{kg}$ tebipenem free base given intravenously (Table 2 and Fig. 4C). 
Table 2

The pharmacokinetics of tebipenem and tebipenem pivoxil

\begin{tabular}{|c|c|c|c|c|c|}
\hline \multirow[t]{2}{*}{ Value } & \multicolumn{3}{|l|}{ Mice } & \multirow{2}{*}{$\begin{array}{l}\text { Piglet } \\
\begin{array}{l}\text { Tebipenem- } \\
\text { Pivoxil PO } \\
(n=2)\end{array}\end{array}$} & \multirow{2}{*}{$\begin{array}{l}\text { Paediatric } \\
\text { population } \\
17\end{array}$} \\
\hline & $\begin{array}{l}\text { Tebipenem } \\
\text { SQ }(n=3)\end{array}$ & $\begin{array}{l}\text { Tebipenem } \\
\text { PO } \\
(n=3)\end{array}$ & $\begin{array}{l}\text { Tebipenem- } \\
\text { Pivoxil PO } \\
(n=3)\end{array}$ & & \\
\hline $\mathrm{C}_{\max }(\mu \mathrm{g} / \mathrm{ml})$ & $71.0 \pm 15.3$ & $3.7 \pm 0.9$ & $61.6 \pm 3.9$ & $9.0 \pm 0.8$ & $7.5 \pm 3.9$ \\
\hline $\begin{array}{l}\mathrm{C}_{\max } \text { Normalized } \\
\left(\mathrm{kg}{ }^{\star} \mu \mathrm{g} / \mathrm{mL} \cdot \mathrm{mg}\right)\end{array}$ & $1.8 \pm 0.4$ & $0.08 \pm 0.02$ & $1.2 \pm 0.08$ & $0.23 \pm 0.08$ & $1.27 \pm 0.69$ \\
\hline $\mathrm{T}_{\max }[$ Range] (hours) & {$\left[\begin{array}{ll}0.25 & 0.25\end{array}\right]$} & {$\left[\begin{array}{ll}0.5 & 1\end{array}\right]$} & {$\left[\begin{array}{lll}0.25 & 0.5\end{array}\right]$} & [2 2] & $0.7 \pm 0.2$ \\
\hline $\mathrm{AUC}_{0-\text { tlast }}\left(\mathrm{h}^{\star} \mu \mathrm{g} / \mathrm{mL}\right)$ & $80.4 \pm 7.7$ & $7.5 \pm 1.5$ & $93.8 \pm 2.7$ & $32.6 \pm 3.9$ & $16.1 \pm 3.3$ \\
\hline $\begin{array}{l}\text { AUC }_{0}-\text { tlast Normalized } \\
\left(h^{\star} \mathrm{kg}^{\star} \mu \mathrm{g} / \mathrm{mL} \cdot \mathrm{mg}\right)\end{array}$ & $2.1 \pm 0.2$ & $0.15 \pm 0.03$ & $1.8 \pm 0.05$ & $0.84 \pm 0.12$ & $2.65 \pm 0.54$ \\
\hline Bioavailability (\%) & $44 \pm 4$ & $4 \pm 1$ & $52 \pm 1$ & ND & ND \\
\hline
\end{tabular}

For the specific treatment of enteric infections, oral administration of tebipenem pivoxil would theoretically facilitate release of the active ingredient at the site of infection. Therefore, we conducted qualitative MALDI-TOF analysis to assess the distribution of tebipenem pivoxil and (hydrolysed) tebipenem free base upon oral administration of tebipenem pivoxil in the intestinal tract of mice. Monitorization of pro-drug and drug at 15 and 60 minutes (Fig. 4A and 4B, respectively) post administration indicated that tebipenem pivoxil could be detected in the intestinal lumen, while tebipenem free base could be detected both in the lumen and within gut enterocytes. These data suggest that tebipenem pivoxil is only partly hydrolysed in the intestinal lumen, but hydrolysed immediately after penetration into enterocytes lining the apical surface of the gut, making it ideal for the treatment of invasive gut bacteria.

To determine if an increased systemic concentration of active drug by orally dosing the pivoxil prodrug resulted in superior performance, we compared the efficacy of the tebipenem pivoxil and tebipenem free base after challenging mice with Shigella. Oral tebipenem pivoxil resulted in a 2.3-log reduction of Shigella in the small intestine compared to no antimicrobial 24 hours post-infection. The quantity of Shigella in the small intestine in mice treated with oral tebipenem pivoxil was significantly lower at the same time point than tebipenem free base (1.2-log reduction, $p \leq 0.05$ ) (Fig. 4D). We observed a similar in reduction in Shigella CFU the large intestine, but the difference in the efficacy between tebipenem pivoxil 
and tebipenem free base (with respect to reduction in Shigella CFU) was not statistically significant (data not shown). Notably, neither formulation reduced the bacterial load in the gut to below detectable limits.

Aiming to exploit a more physiological Shigella infection model we also measured the efficacy of tebipenem pivoxil in gnotobiotic piglets using a $50 \mathrm{mg} / \mathrm{Kg}$ dose of tebipenem pivoxil (equivalent to the licenced $6 \mathrm{mg} / \mathrm{kg}$ dose in Japan). The tebipenem Cmax in the piglet was $8 \mu \mathrm{g} / \mathrm{ml}$ and equivalent to those reported in a paediatric population ${ }^{17}$; over the course of six hours the concentration of tebipenem declined to $1 \mu \mathrm{g} / \mathrm{ml}$ (Fig. 5A). Tebipenem pivoxil reduced the frequency of diarrheal stools in infected piglets, performing in an equivalent fashion to the ciprofloxacin control. Explicitly, tebipenem pivoxil was highly efficacious in reducing Shigella in the gut, with a single dose of tebipenem pivoxil 24 hours after infection reducing the CFU/g Shigella in stool from $1 \times 10^{7}$ to zero within 24 hours, again performing equally well as ciprofloxacin (Fig. 5B). We lastly performed necropsy and histopathology of the large intestine in tebipenem pivoxil- and placebo-treated piglets in order to compare the presence of intestinal lesions, inflammation, and the presence of intracellular Shigella. The histopathology showed a marked reduction in intestinal lesions, and inflammation between the placebo-treated group and the group who received tebipenem pivoxil; no viable Shigella could be observed in section from the treated large intestine (Fig. 5C and 5D).

\section{Discussion}

AMR poses one of the greatest challenges in global health; therefore, new treatments and approaches are urgently needed to treat infections caused by antimicrobial resistant organisms. Here, we exploited a drug screening strategy to identify compounds that may have utility against highly antimicrobial resistant enteric pathogens, specifically MDR/XDR Shigella. Ultimately, we identified and progressed tebipenem pivoxil (a licenced oral carbapenem) to pre-clinical testing for Shigella infections. Tebipenem pivoxil has many suitable characteristics for the treatment of infections caused by MDR and XDR Gram-negative bacteria; it is broad spectrum, it is rapidly bactericidal, is already used in paediatric clinical practice, and it can be administered orally, making it highly suited for the treatment of gastrointestinal infections in the community. Despite these positives, there are potential issues surrounding the widespread use of this drug, most notably the potential to generate resistance against carbapenems, a very important last resort class of antibacterial compounds.

We initiated the project by screening a large compound library against a single Shigella isolate grown extracellularly. Notably, none of the chemicals meeting the desired criteria (inhibition of bacterial growth with a physicochemical and safety profile suitable for downstream developability) represented novel chemical classes. All hits were already known to possess antibacterial properties and belonged to either the GSK antibacterial subset or were commercially available. The fact that no new modes of action were identified after screening 1.7 million compounds via extracellular in vitro screening is concerning, suggesting we are close to exhausting the chemical space for antibacterial agents. However, innovative phenotypic assays, which interrogate other stages of the bacterial infection, such as intracellular activity or invasion blockade, may offer new opportunities for developing narrower spectrum drugs. Using the 
selected approach, we opted to focus on a defined set of compounds with known antibacterial activity against $E$. coli, allowing us to identify two main chemical classes that additionally exhibited activity against clinical Shigella isolates. Specifically, we found that carbapenems had high potency against both laboratory Shigella strains and clinical isolates of MDR Shigella from highly endemic LMICs. Consequently, tebipenem was selected as a lead candidate for further pre-clinical characterization due to the great potency and the availability of a prodrug which allows oral administration.

Tebipenem had enhanced activity (with respect to ciprofloxacin) against a panel of 81 Shigella clinical isolates from Southeast Asia, an observation in line with the high prevalence of ciprofloxacin resistance in many Shigella sonnei and a high proportion of Gram-negative bacterial pathogens across Southeast Asia. Tebipenem additionally exhibited high level of potency against a broad panel of $>300$ clinically relevant MDR enteric pathogens. These data outline the potential use of this antimicrobial for the treatment of enteric infections of suspected bacterial origin without the necessity of an accompanying diagnostic test, which is a common practice in many LMICs. It is noteworthy that carbapenem resistance is emerging in some Gram-negative organisms in Asia and has spread to other regions. This phenomenon is partly associated with the spread of the NDM-1 gene, which encodes a betametalloprotease. Carbapenem resistance has not, as yet, been reported in any Shigella species and is not currently common in other Gram-negative bacteria causing diarrhoea. However, given the propensity of Shigella to develop resistance to commonly used antimicrobials through the acquisition of AMR plasmids and individual gene cassettes, we additionally need to consider the requirement of a companion diagnostic to avoid treatment failures or overemployment of the drug, which my trigger resistance.

We considered that the emergence of resistance against tebipenem (and other carbapenems) may be mitigated through combining tebipenem with drugs with alternative other modes of action, including existing standard of care antimicrobials. Azithromycin, which reduced the MIC for Shigella isolates for tebipenem when tested in combination, offers an encouraging primary combination option, given the common use of azithromycin for the treatment of severe diarrhoea. Additionally, a second potential combination showing in vitro synergy was identified with an LpxC inhibitor, which again showed good activity when tested against the majority of enteric bacteria studied. Again, this observation is encouraging and adds further support to LpxC development programs and may provide a solution for limiting spontaneous resistance against this newer class of antimicrobials. Our data highlight the utility of tebipenem for treating a spectrum of enteric pathogens and the potential benefit of co-administration with recommended antimicrobials suggest that combination therapy with tebipenem is clearly worth exploring through clinical trials and/or through controlled human infection studies with Shigella. The existing safety profile of these drugs opens the possibility of direct progression to a clinical proof of concept study as soon as possible.

Encouragingly, tebipenem pivoxil is approved and marketed in Japan as Orapenem (Meiji Seika) for the treatment of adult and paediatric respiratory tract infections ${ }^{18}$. In addition, Spero Therapeutics are working on a novel formulation with extended half-life for administration in adults which is under clinical evaluation for recurrent urinary tract infections ${ }^{19,20}$. The MICs of the panel of enteric bacteria against 
tebipenem were substantially below the clinical breakpoints reported for efficacious treatment of respiratory tract infections. Shigella infections have a pathophysiology which is distinct to that of respiratory tract infections, but our MALDI-TOF data suggests that tebipenem reaches a high concentration in the enterocytes lining the gastrointestinal surface. We hypothesise that the currently approved dose of Orapenem will reach a higher local concentration in the Shigella infection-relevant cells in the gut than in the respiratory tract. Therefore, the drug distribution behaviour of Orapenem is especially convenient for the treatment of enteric infections, as the active component is specifically released at the site of infection. The release of free tebipenem intracellularly would clear intracellular bacteria (vital for Shigella and Salmonella) and active tebipenem in the intestinal lumen would reduce extracellular bacteria and limit cellular invasion. The animal model data implies this process is occurring, as the drug was highly potent after Shigella challenge and able to clear the gut of infecting organisms with 24 hours of administration in gnotobiotic piglets.

In conclusion, we performed a screening study to identify novel compounds with antibacterial activity against MDR/XDR Shigella. We found that tebipenem had excellent in vitro profile against MDR/XDR Shigella and other Gram-negative bacteria; the oral bioavailability of tebipenem pivoxil makes it ideal for the treatment of gastrointestinal infections. Experimentation in animal models of shigellosis found the drug to be highly efficacious with activity comparable to ciprofloxacin, when animals were challenged with ciprofloxacin susceptible Shigella isolates. We are in desperate need of alternative antimicrobials for infections caused by MDR/XDR bacteria and our data outline the oral carbapenem tebipenem as an ideal repurposing candidate. The testing and controlled introduction of tebipenem pivoxil combinations for MDR Shigella infections may slow the rate of spread of organisms that exhibit resistance to current standard of care antimicrobials or provide an extended window of opportunity for the further development and introduction of Shigella vaccines.

\section{Materials And Methods}

\section{Reagents and compounds}

All commercially available chemicals were purchased from either Sigma Aldrich, UK, AK Scientific, or Fischer Chemicals. The GSK 1.7 million compound screening set was sub-selected from the 8 million compound GSK collection using the principles described by Harper and co-workers ${ }^{21}$. Background of the structural filters or property constraints developed to create a "clean" set of compounds with regard to compound quality (purity/surety), no undesirable/reactive chemotypes and in the appropriate property space. This process has been described previously by Chakravorty and co-workers ${ }^{22}$. The set of 4,000 compounds with known antibacterial pedigree from the GSK antibacterial programs was selected from GSK compound collection using an MIC $<1 \mu \mathrm{M}$ against $E$. coli as cut-off value for selection.

\section{Organisms and growth conditions}

For the initial screening we used Shigella flexneri 2457T (ATCC 700930). Organisms were grown at $37^{\circ} \mathrm{C}$ in Mueller Hinton medium overnight (Sigma Aldrich, UK). Hits from the initial screening were then profiled 
in two Shigella clinical isolates originating from Ho Chi Minh City, Vietnam: S. flexneri EG478 and S. sonnei 02-1181. Selected compounds were profiled in a panel containing 338 clinical isolates of enteric pathogens containing Shigella (Vietnam and Kenya; $\mathrm{n}=135$ ), non-typhoidal Salmonella (Vietnam; $\mathrm{n}=$ 117), typhoidal Salmonella (Vietnam and Nepal; $n=4$ ) Campylobacter (Vietnam; $n=75$ ), and various pathogenic E. coli species (Vietnam; $n=7$ ). Campylobacter isolates were grown in 2.5\% Laked Horse Blood cation-adjusted Mueller Hinton medium (CAMHB, Sigma Aldrich) and incubated in microaerophilic conditions $\left(10 \% \mathrm{CO}_{2}, 5 \% \mathrm{O}_{2}\right.$ and $\left.85 \% \mathrm{~N}_{2}\right)$ at $42^{\circ} \mathrm{C}{ }^{23}$. Other clinical strains were grown in CAMHB medium at $37^{\circ} \mathrm{C}$. The antimicrobial susceptibility profile of these organisms is described included in Supplementary Table 1.

\section{High throughput in-vitro Shigella growth inhibition assay}

An extracellular assay adapted from Franzblau et $a^{24}$. was used to determine the IC50 and the IC90 of potential inhibitors of Shigella flexneri ATCC 2457T. The assay measures the effect of compounds on the bacteria using resazurin reduction (Thermo Fisher Scientific, UK) as a surrogate of bacterial viability. Prior to day of experimentation, an overnight bacterial culture was inoculated 1/100 in Mueller Hinton medium and incubated for 24 hours; the bacterial culture was inoculated 1/5 into fresh Mueller Hinton medium. Bacterial growth was measured at $600 \mathrm{~nm}$ after three hours growth (expected OD 0.4-0.6) and referenced to the corresponding $\mathrm{CFU} / \mathrm{mL}$. The bacterial inoculum was diluted $1 \times 10^{6} \mathrm{CFU} / \mathrm{mL}$ to perform the assay. Subsequently, 1,536-well polystyrene assay plates (HiBase, $\mu$ Clear, Greiner Bio-One) were prepared by dispensing $50 \mu \mathrm{l}$ of compound from master plates at $1 \mathrm{mM}$ in each well using a LabCyte Echo system. The final assay volume was $5 \mu \mathrm{l}$ and final compound concentration was $10 \mu \mathrm{M}$. The 11th and 12th columns were positive growth controls containing $50 \mathrm{~nL}$ of DMSO. The 34th and 35th columns contained $50 \mathrm{~nL}$ of Moxifloxacin at $2 \mathrm{mM}$ as negative growth control. $5 \mu \mathrm{l}$ of bacterial inoculum was dispensed into compound pre-dispensed plates using a Multidrop Combi dispenser (Thermo Scientific). Plates were agitated for 10 seconds to ensure mixing and incubated at $37^{\circ} \mathrm{C}$ overnight (16-17 hours). Following overnight culture, $2 \mu$ resazurin solution (Resazurin tablets for milk testing, $\mathrm{BDH}$ ) was added to each well and incubated in at ambient temperature in the dark for 3 hours. Fluorescence intensity was measured using an Envision plate reader (PerkinElmer). For IC50 and IC90 determination, data were normalized by using DMSO only as positive control (100\% bacterial growth) and $20 \mu \mathrm{M}$ moxifloxacin as negative control ( $0 \%$ bacterial growth). Data were analysed using Base software and statistical cut-off analysis was performed for hit selection following GSK standard protocol. The percentage growth inhibition was calculated per well according to the plate controls. These values were aggregated to calculate the robust mean and standard deviation (SD) of the distribution. The robust mean was calculated starting from the median and the MAD of the distribution with an iterative process that aimed to remove outliers. Once the mean and SD were known, all compounds above mean + 3SD were selected as positive hits. After compound analysis and removal of structures with alerts, prioritized compounds were progressed to dose-response analysis. To perform the dose-response curves, compounds were diluted in DMSO at 1:3 serial dilutions in concentrations from 100 to $0.00169 \mu \mathrm{M}$.

\section{Growth inhibition assays with clinical isolates}


Compound screening and dose response assays have been described previously ${ }^{25,26}$. All chemical testing was performed at an initial concentration of $10 \mu \mathrm{M}$. The bacteria selected for screening were cultured on the Luria-Bertani agar overnight and their colonies would be separately mixed in sterile PBS to form 0.5 McFarland solutions. The prepared bacterial suspensions were then evenly spread on MuellerHinton agar by sterile cotton swabs (Medical Wire - MWE). To test the chemicals, $5 \mu$ l of the $10 \mu \mathrm{M}$ solutions were pipetted onto the surface of the media; and $5 \mu$ of $10 \mu \mathrm{M}$ of ciprofloxacin/meropenem was used as a positive control and sterile PBS as a negative control. Plates were incubated overnight at $37^{\circ} \mathrm{C}$. For the screen, the activity of the chemical was recorded as being active (yes) or non-active (no) for each of the tested chemicals. A non-active result was recorded when there was no evidence of bacterial inhibition and the culture area was indistinguishable from the negative control. An active result was recorded when there was an obvious zone of inhibition of bacterial growth and the observed zone was similar to that of the positive control, i.e. an area without bacterial growth where the media could be observed.

Minimum inhibitory concentration test (Micro dilution assay): The MIC of chemicals is tested with $1 \mathrm{mM}$ chemical stock solution, $\mathrm{MH}$ broth and $5 \times 10^{5} \mathrm{CFU} / \mathrm{ml}$ bacteria (final concentration). Preparing the twofold serial dilution in 96-well plate, the experiment starts with $10 \mathrm{uM}$ chemical in the first column and will be further diluted 2 -fold to the next column until column 8 . The bacteria were added and incubated at $37^{\circ} \mathrm{C}$ overnight. After that, $10 \mathrm{ul}$ of each well is drop on a Nutrient agar Petri plate for detection of bacterial growth and the plates are incubated overnight at $37^{\circ} \mathrm{C}$. Results are interpreted as minimal concentration necessary to inhibit growth.

Time-kill curve assays were performed in 50ml-tubes by culturing Shigella spp. in CAMHB medium in the presence of four antimicrobial concentrations in doubling dilutions ranging from $0.5 x \mathrm{MIC}$ to $4 \mathrm{xMIC}$. The MICs were determined before the experiment using the broth micro-dilution method. Bacterial stocks were prepared in $\mathrm{NaCl} 0.9 \%$ and added into each tube to obtain a concentration of $5 \times 10^{5} \mathrm{CFU} / \mathrm{mL}$. Bacteria were grown with agitation at $200 \mathrm{rpm}$ at $37^{\circ} \mathrm{C}$ and monitored over a time-course of 24 hours $(0,2,4,6,8$, and 24 hours). For every concentration and time point, bacterial cultures were diluted and inoculated onto Nutrient Agar (NA, Oxoid). NA plates were incubated at $37^{\circ} \mathrm{C}$ overnight and bacterial colonies were enumerated.

\section{Combination studies}

Before initiating the drug combination assays, the MIC values for both compounds were determined against ATCC organisms (as previously described) to design the checkerboard layout. The standard checkerboard titration method based on isobolograms was used to study the in vitro interaction between tebipenem and other compounds (WHO recommended treatments and an LpxC inhibitor). 20uM moxifloxacin was used as negative control (100\% bacterial growth inhibition) in one column and DMSO as positive control (100\% bacterial growth). $25 \mu \mathrm{l}$ of $1 \times 10^{6} \mathrm{CFU} / \mathrm{mL}$ bacterial culture was added to each well of 384-well plates ( $\mu$ Clear; Greiner Bio-One). Plates were incubated overnight at $37^{\circ} \mathrm{C}$ and $10 \mu \mathrm{l}$ of resazurin were added. Plates were incubated at ambient temperature for three hours. Bacterial growth 
inhibition was measured by fluorescence in a Envision plate reader (PerkinElmer). Data were managed in Microsoft Excel and analysed in Grafit 7 (Erithacus) data analysis software.

Combination studies with clinical strains were performed in 96-well plates (Greiner) following the same checkboard titration method in a final volume of $200 \mu \mathrm{l}$ in each well. The range of final working concentrations of each antimicrobial varied and depended on the MIC of each selected strain.

MICs were determined for drug A and drug B alone and in combination. The MIC of both drugs in combination were expressed as fractions of the MIC of the drug alone normalized to 1 , which represents the Fractional Inhibitory Concentration (FIC). The sum of FIC was expressed using the following equation: (MIC of drug A in combination / MIC of drug A alone) + (MIC of drug B in combination / MIC of drug B alone) $27-30$.

\section{Spontaneous resistant mutants}

The frequency of spontaneous resistant mutants (FoR) was determined for tebipenem. Moxifloxacin, ciprofloxacin, and ampicillin were used as internal controls; bacterial inoculum without drug was used a as positive control. The bacteria inoculum $\left(1 \times 10^{5} \mathrm{CFU} / \mathrm{mL}\right)$ for the assay was prepared by diluting a log phase incubated at $37^{\circ} \mathrm{C}$ for $3-4$ hours. Mueller Hinton agar was prepared containing $4 \times \mathrm{MIC}$ and $10 \mathrm{xMIC}$ and inoculated with different concentrations of bacteria $\left(1 \times 10^{9}, 1 \times 10^{8}\right.$, and $\left.1 \times 10^{7} \mathrm{CFU} / \mathrm{mL}\right)$. Plates were incubated overnight at $37^{\circ} \mathrm{C}$. The frequency of spontaneous resistant mutants was calculated by using the formula resistant CFUs/inoculum CFUs.

\section{Extrapolation of efficacy doses from Orapenem approved treatment in paediatric population}

Pharmacokinetic parameters of Orapenem in paediatric population at the approved doses $(4-6 \mathrm{mg} / \mathrm{kg}$, bid) were obtained from Sato et al. and pharmacokinetic parameters in rats were generated in house ${ }^{17}$. Information regarding Orapenem oral bioavailability was extracted from Kijima et al. ${ }^{17}$. For the calculation of tebipenem pharmacokinetic behaviour in different animal species (i.e. mouse and piglet) conserved intrinsic clearance $\left(\mathrm{CL}_{\text {int }}\right)$ and distribution volume (Vss) across the different animal species have been assumed. A mono-compartmental PK model has been used for PK simulation using matlab simbiology (2019b) software. The equivalent doses in the different animal species were calculated using tebipenem free drug $\mathrm{t} / \mathrm{MIC}$ as comparable parameter.

\section{In vivo pharmacokinetic analysis}

All mice experiments were performed in female C57 mice obtained from Charles River Laboratories, (Wilmington, MA) and housed in cages in groups of three animals with water and food ad libitum. Animals were acclimated for seven days prior to infection. At the beginning of the experiment the mice weight was 20-30 mg. Tebipenem was dissolved in 20\% Encapsine (Sigma Aldrich), 5\% DMSO (Sigma Aldrich) in saline solution (Sigma Aldrich) at $0.5 \mathrm{mg} / \mathrm{ml}$ for intravenous administration and in $1 \%$ Methylcellulose (Sigma Aldrich) in water at $5 \mathrm{mg} / \mathrm{ml}$ for oral administration. Tebipenem pivoxil was dissolved in $1 \%$ Methylcellulose (Sigma Aldrich) in water at $5 \mathrm{mg} / \mathrm{ml}$ for oral administration. 
For PK analysis, $15 \mu \mathrm{l}$ of tail blood were collected by microsampling at $0.08,0.25,0.5,1,2,4,6$, and 24 hours for intravenous pharmacokinetic and $0.25,0.5,0.75,1,2,4,6$, and 24 hours for oral pharmacokinetics. Blood samples $(15 \mu \mathrm{l})$ were mixed and vortexed with $30 \mu \mathrm{l}$ sterile water and snap frozen at $-80^{\circ} \mathrm{C}$ prior to analysis. Blood samples were thawed at ambient temperature and $10 \mu \mathrm{L}$ were mixed with ACN:MeOH (80:20), filtered ( $0.2 \mu \mathrm{m}$ filter plate, Whatman) and analysed by LCMS.

\section{Drug distribution studies (intestine homogenate and MALDI analysis)}

To determine the distribution of tebipenem pivoxil and free base in the different parts of the intestine, tebipenem pivoxil was orally administrated at $5 \mathrm{mg} / \mathrm{ml}$ to $\mathrm{C} 57$ mice $(\mathrm{n}=12$, target dose $50 \mathrm{mg} / \mathrm{kg})$ in four groups of three animals. Animals were euthanized at $0.25,0.5,1$, and 4 hours and different sections of the intestine (duodenum, jejunum, ileum, caecum and colon) were collected and frozen at $80^{\circ} \mathrm{C}$. For analysis of intestine homogenate, samples were thawed at ambient temperature, mixed with water (1:2) and homogenized during $10 \mathrm{seg}$ by turrax. Subsequently, $20 \mu \mathrm{L}$ of homogenate were mixed with $200 \mu \mathrm{l}$ of ACN:MeOH (80:20) and filtered using $0.2 \mu \mathrm{m}$ filter plate (Whatman) for LCMS analysis. For MALDI imaging, $50 \mathrm{~mm}$ of the central part of the intestine were mounted in a sample disc on dry ice with a solution of $1 \%$ methylcellulose (Sigma Aldrich, UK). $12 \mu \mathrm{m}$ thick tissue (sagital sections) were obtained using a cryostat (Leica CM3600; Leica Microsystems Inc., Wetzlar, Germany) at $-20^{\circ} \mathrm{C}$ and collected in conductive-coated slides for MALDI Imaging (Bruker Daltonic). After the "MALDI" section was obtained a consecutive section for histology was taken. Prior to matrix coating samples for MALDI were optical scanned in a PathScanner. The matrix deposition was conducted in a sprayer ImagePrep System (Bruker Daltonic) which use vibrational vaporization technology. The matric solution was $\alpha-\mathrm{CHCA}$ (a-Cyano-4hydroxycinnamic acid (Sigma Aldrich) dissolved in 70:30 methanol/water (v/v) containing $0.2 \%$ TFA at 7 $\mathrm{mg} / \mathrm{mL}$ as the matrix solution. MALDI MSI data were obtained using a Bruker UltrafleXtream MALDI TOF/TOF (Bruker Daltonic, Germany)

\section{LC-MS analysis}

An acquity ultra-performance liquid chromatography (UPLC) system (Waters Corp., Milford, MA, USA) coupled to a triple quadrupole mass spectrometer (API 4000 ${ }^{\mathrm{T}}$, AB Sciex, Foster City, CA, USA) was used for LC-MS analysis of tebipenem free base in the PK blood samples. The chromatographic separation was performed at $0.4 \mathrm{ml} / \mathrm{min}$ in an Acquity UPLC ${ }^{\mathrm{m}}$ BEH C18 column $(50 \times 2.1 \mathrm{~mm}$ i.d., $1.7 \mathrm{~mm}$; Waters Corp.) at $40^{\circ} \mathrm{C}$ with Acetonitrile (Sigma Aldrich) and $0.1 \%$ formic acid as eluents.

\section{In vivo mouse efficacy studies}

The S. flexneri lux 1 strain was used to infect mice for the mouse efficacy experiments. S. flexneri lux1 expresses a bacterial luciferase reporter as previously described and was created from S. flexneri M90T Sm (ATCC BAA-2402) purchased from the American Type Culture Collection (ATCC, Manassas, VA). Broth cultures of $S$. flexneri strain lux 1 were grown at $37^{\circ} \mathrm{C}$ in Trypticase soy broth (TSB) with agitation at 220 rpm. For solid media cultures of S. flexneri strain lux1, an agar form of Trypticase soy broth with the addition of $0.01 \%$ Congo Red. $30 \mu \mathrm{g} / \mathrm{mL}$ streptomycin was used for selection of the lux 1 strain and $0.1 \%$ sodium deoxycholate was added to the broth to promote expression of proteins involved in Shigella cellular invasion ${ }^{31}$. 
The in vivo efficacy of anti-Shigella drug candidates was evaluated using a previously published mouse model of shigellosis ${ }^{31}$. In brief, an overnight $S$. flexneri lux 1 broth culture was diluted 1:100 and grown to log-phase (3-4 hours). Log-phase bacteria $\left(\mathrm{OD}_{600} \sim 0.4\right)$ were centrifuged, and the resulting pellet was washed in DPBS without calcium and magnesium (Thermo Fisher Scientific). Bacteria were centrifuged again, and pellet was resuspended in DPBS to the target concentration of $5 \times 10^{7}$ S. flexneri/200 $\mu \mathrm{L}(10$ $\mathrm{mL} / \mathrm{kg}$ ). Each dose of bacteria was confirmed with luminescence using an EnVision Plate Reader (Perkin Elmer) for S. flexneri lux1.

Female B6 mice (000664 Black 6, Jackson Laboratories) aged 9-10 weeks were weighed and infected IP with $5 \times 10^{7} \mathrm{CFU} /$ mouse (total $\mathrm{n}=7-10$ per group). Antimicrobials were suspended in oral vehicle $(1 \%$ carboxymethyl cellulose), and mice were administered antimicrobials or vehicle control PO at 2 and 16 hours post infection (p.i.). Ciprofloxacin was used as positive control and administered at $40 \mathrm{mg} / \mathrm{kg}$ of body weight. Mice were euthanized 24 hours p.i. and the small intestine and cecum/large intestine were collected. Gastrointestinal tissue was weighed, resuspended in $1 \mathrm{~g} / \mathrm{mL}$ DPBS, homogenized, and plated on trypticase soy agar plates with $30 \mu \mathrm{g} / \mathrm{mL}$ streptomycin to quantify $S$. flexneri CFUs.

\section{In vivo gnotobiotic piglet studies}

To test the efficacy of tebipenem for treating Shigella in vivo we exploited gnotobiotic piglet model. The gnotobiotic piglet model is the closest surrogate of severe diarrhoea caused by Shigella infection because animals develop diarrhoea upon oral bacterial challenge, without any additional interventions (i.e. immunosuppression, diet modification, or antimicrobials). This model has been exploited previously in several Shigella vaccine development studies ${ }^{32}$. For the purposes of this study a litter of 12 piglets was used. Animals were delivered by Caesarean section and held in sterile isolators for the entry of the study. Healthy animals were infected with $1 \times 10^{9} \mathrm{CFU}$ of Shigella 24 hours after birth; drug/placebo were administered 24 hours after infection. Four piglets received Tebipenem pivoxil ( $50 \mathrm{mg} / \mathrm{kg}$ ) every 12 hours

for 5 days, 4 piglets received formulation vehicle (1\% methyl cellulose) every 12 hours for 5 days (infection control), 2 piglets received ciprofloxacin $(40 \mathrm{mg} / \mathrm{kg}$ ) every 12 hours for 5 days (treatment control), 2 uninfected piglets were administered Tebipenem pivoxil $(50 \mathrm{mg} / \mathrm{kg}$ ) every 12 hours for 5 days, (to assess treatment side effects). Efficacy was determined by measuring bacterial count in faeces and time to cessation of symptoms.

\section{Declarations}

\section{Ethics approval and consent to participate}

All studies were conducted in accordance with the European Directive 2010/63/EEC and the GSK Policy on the Care, Welfare and Treatment of Laboratory Animals or were reviewed by the Institutional Animal Care and Use Committee at the institution where the work was performed. 
Consent for publication was incorporated as a component of entrance into the study.

Availability of data and materials

All data are presented in the manuscript and raw data are freely available upon request from the corresponding author.

Competing interests

The authors declare no competing interests.

\section{Funding}

We thank the Tres Cantos Open Lab Foundation (projects TC239 and TC246) and the Bill and Melinda Gates Foundation (grant OPP1172483) for financial support. Stephen Baker is supported by a Wellcome senior research fellowship (215515/Z/19/Z). The funders had no role in the design and conduct of the study; collection, management, analysis, and interpretation of the data; preparation, review, or approval of the manuscript; and decision to submit the manuscript for publication.

\section{Authors' contributions}

Conceptualization: EFA, LB, SB

Formal analysis: EFA, PVV, AGP, SLMA, SB

Provided samples: PVV, NTDH, THT, DD, TS

Methodology: EFA, PVV, CdC, BU, AC, AP, NTDH, THT, MM, SS, DD, JdM, AGP, BS, PP, JW, MSM, SLMA, TS

Writing original draft: EFA, PVV, AGP, LB, SB

Review and editing: EFA, PVV, AGP, LB, SB

Read and approved final version of manuscript: EFA, PVV, CdC, BU, AC, AP, NTDH, THT, MM, SS, DD, JdM, AGP, BS, PP, JW, MSM, SLMA, TS, LB, SB

\section{Acknowledgements}

We would like to thank Matthew Hulverson and Shareef Shaheen from Washington University for their contribution in the development of the mouse model of shigellosis. We thank Pablo Castañeda and Janneth Fatima Indira Rodrigues from GSK for their scientific advice during fruitful discussions. We would like to thank the In Vivo Study Delivery team (IVSD) from GSK Tres Cantos for their essential contribution to the $\mathrm{PK}$ in vivo experiments.

\section{References}


1. Theuretzbacher, U. Curr. Opin. Microbiol. 39, (2017).

2. Kotloff, K. L. Pediatr. Clin. North Am. 64, (2017).

3. Khalil, I. A. et al. Lancet. Infect. Dis. 18, (2018).

4. Kotloff, K. L. et al. Lancet 382, 209-222 (2013).

5. Liu, J. et al. Lancet 388, 1291-301 (2016).

6. Kotloff, K. L., Riddle, M.S., Platts-Mills, J. A., Pavlinac, P., Zaidi, A. K. M. Lancet 391 801-812, (2018).

7. Thanh Duy, P. et al. Nature Microbiology 5, 256-264 (2020).

8. Chung The, H. et al. Nat. Commun. 10, (2019).

9. Baker, K. S. et al. Lancet Infect. Dis. 15, 913-921 (2015).

10. WHO. https://www.who.int/news-room/detail/27-02-2017-who-publishes-list-of-bacteria-for-whichnew-antibiotics-are-urgently-needed. (Accessed: 25th September 2020)

11. Gwynn, M.N., et al. Ann. N. Y. Acad. Sci. 1213, (2010).

12. Gamo, F. J., et al. Nature 465, (2010).

13. Ballell, L., et al. ChemMedChem 8, (2013).

14. Ballell, L., et al. Nat. Rev. Drug Discov. 15, (2016).

15. Jain, A., et al. Expert Rev. Anti. Infect. Ther. 16, (2018).

16. García-Quintanilla, M. et al. Antimicrob. Agents Chemother. 60, (2016).

17. Sato, N. et al.. Drug Metab. Pharmacokinet. 23, (2008).

18. Kataoka, H., Kasahara, H., Sasagawa, Y., Matsumoto, S. S. Jpn J Antibiot 53-76 (2016).

19. Arends, S. J. R. et al. Antimicrob. Agents Chemother. 63, (2019).

20. Eckburg, P. B., et al. Antimicrob. Agents Chemother. 63, (2019).

21. Harper, G., Pickett, S. D., Green, D. V. Comb. Chem. High Throughput Screen. 7, (2004).

22. Chakravorty, S. J. et al. SLAS Discov. Adv. life Sci. R D 23, (2018).

23. CLSI. CLSI Document M100-S25 32, (2014).

24. Franzblau, S. G., et al. J. Clin. Microbiol. 36, (1998).

25. Van Voorhis, W. C., et al. PLoS Pathog. 12, (2016).

26. Wiegand, I., Hilpert, K., Hancock, R. E. Nat. Protoc. 3, (2008).

27. Le Minh, V., et al. J. Med. Microbiol. 64, (2015).

28. Petersen, P. J., Labthavikul, P., Jones, C. H., Bradford, P. A. J. Antimicrob. Chemother. 57, (2006).

29. Hall, M. J., Middleton, R. F., Westmacott, D. J. Antimicrob. Chemother. 11, (1983).

30. EUCAST. Clin. Microbiol. Infect. 6, (2000).

31. McCloskey, M. C., et al. Sci. Rep. 9, (2019).

32. Jeong, K. I., Venkatesan, M. M., Barnoy, S., Tzipori, S. Vaccine 31, (2013).

\section{Figures}


A

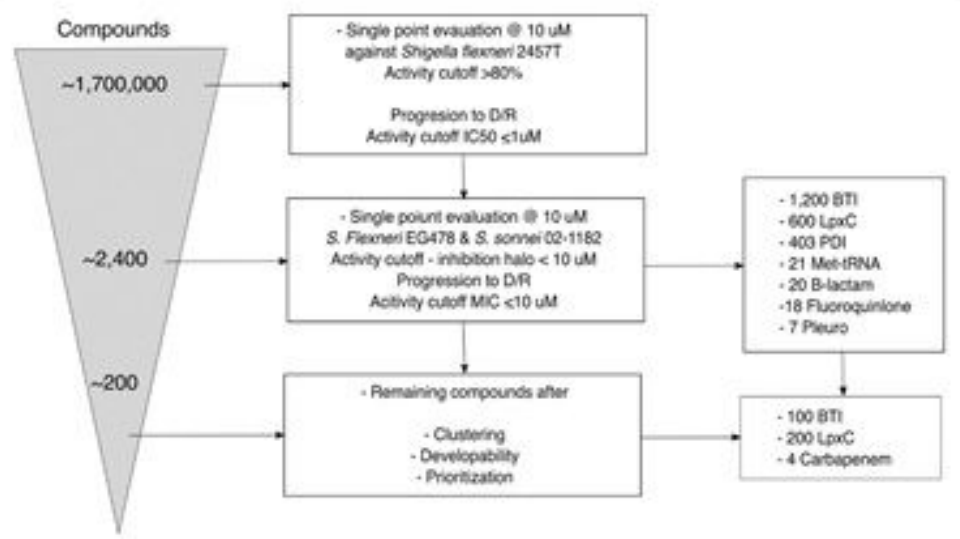

B

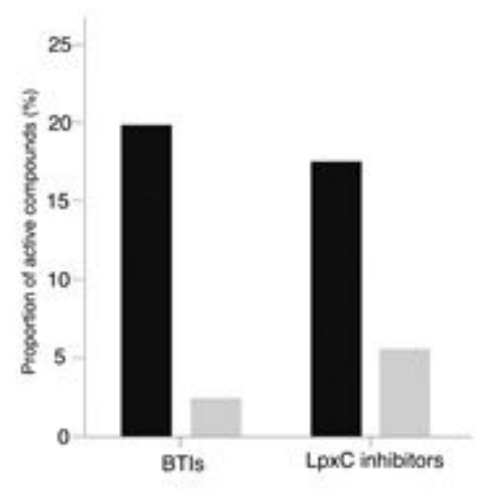

\section{Figure 1}

Screening compounds for antibacterial activity against Shigella isolates A) Flowchart outlining the screening process in three key stages 1 : identification of compounds with antibacterial activity against $\mathrm{S}$. flexneri 2457T from an initial set of $\sim 1.7$ million chemicals at a concentration of $10 \mu \mathrm{M}$. 2: identification of compounds that have antibacterial activity against S. flexneri $2457 T$ and S. sonnei 02-1181 (clinical isolates from Vietnam) from an active subset of 2,269 compounds in 7 classes at a concentration of 10 $\mu \mathrm{M}$. 3: Selection of compounds that were suitable for further progression after selecting for clustering, developability and prioritization, resulting in $\mathrm{x}$ compounds in 3 classes. B) Bar charts showing the activity profile (proportion of chemicals with antibacterial activity) of BTIs and LpxC inhibitors against the S. flexneri 2457T laboratory isolate (black) and the two Vietnam clinical isolates (S. flexneri 2457T and S. sonnei 02-1181; grey). 
A

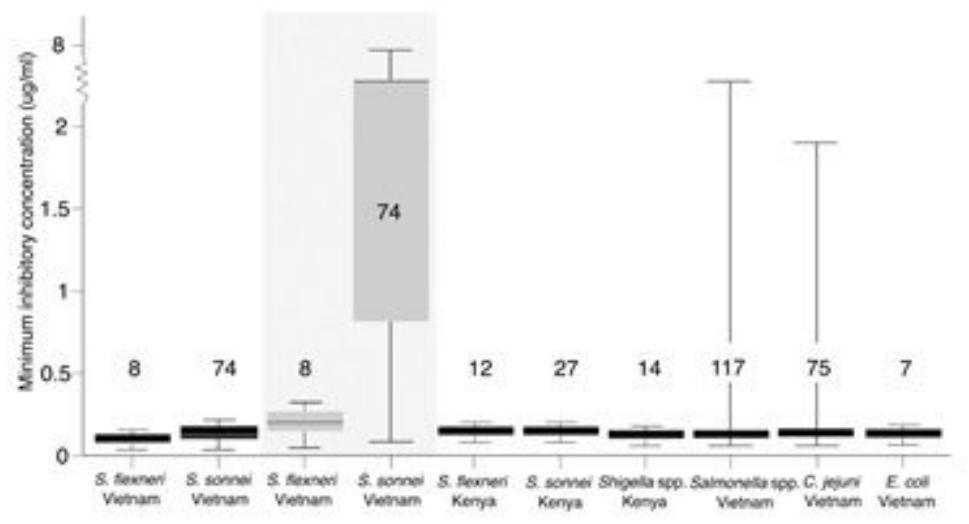

B

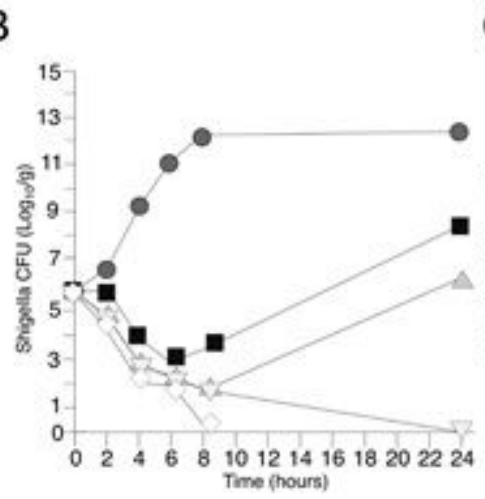

$\mathrm{C}$

$\mathrm{D}$
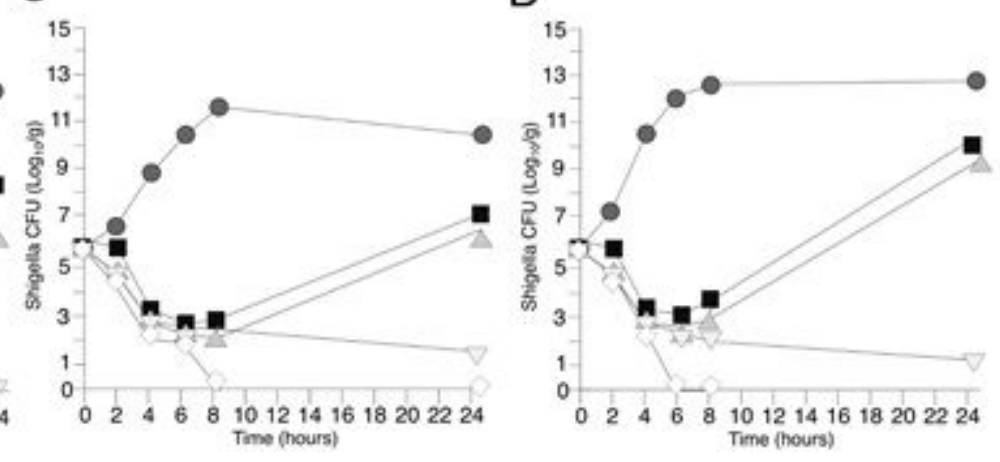

Figure 2

The in vitro activity tebipenem against various enteric pathogens $A$ ) Boxplots of the minimum inhibitory concentrations ( $\mathrm{MIC} ; \mu \mathrm{g} / \mathrm{ml}$ ) of tebipenem (black boxes) and ciprofloxacin (grey boxes) of clinical isolates of Shigella spp. from Vietnam and Kenya and a range of other enteric pathogens. The boxes show the interquartile range of MICs (median line) and whiskers show the highest and lowest MICs; number of isolates screened are highlighted. Time kill curves of $0 x$ (grey circles), $0.5 x$ (black squares), $1 x$ (grey triangles), 2x (light grey inverted triangles), and 4x (white diamonds) MIC tebipenem over 24 hours against B) S. flexneri EG478, C) S. flexneri_01_0417, and D) S. sonnei_02_1181; counts shown in CFU/ml. 
A

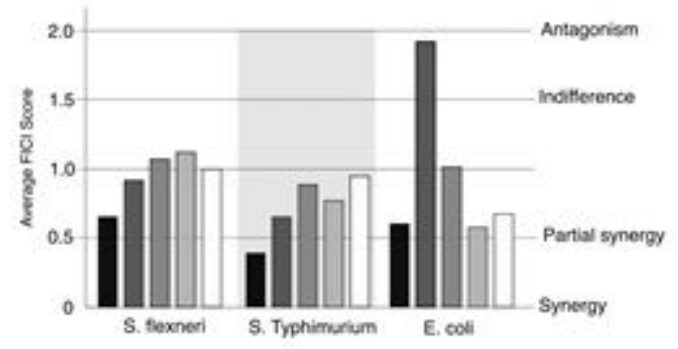

B

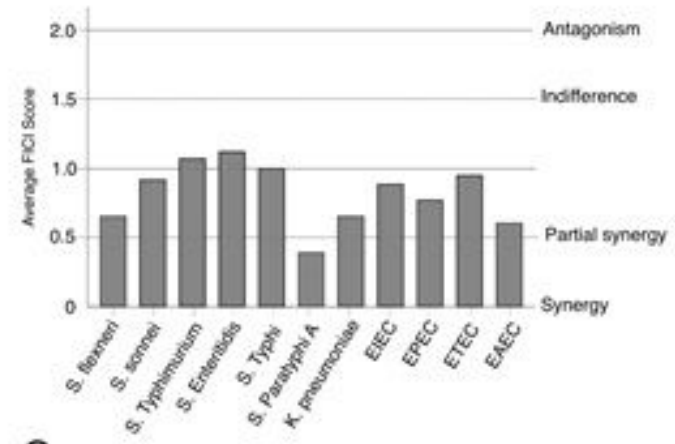

C

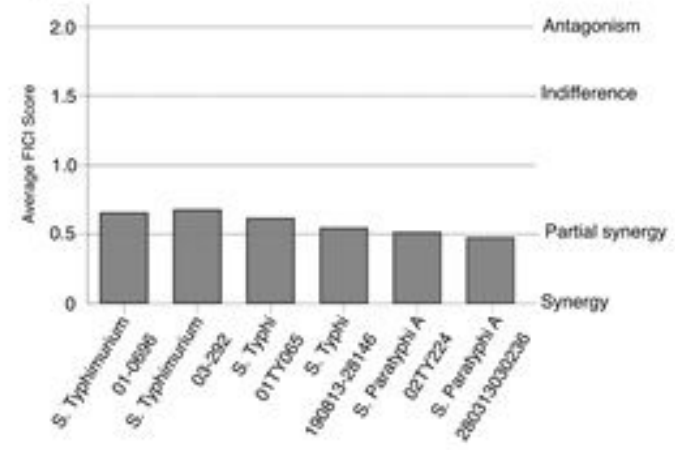

Figure 3

In vitro synergy of tebipenem with standard of care antimicrobials A) Bar chart showing the average $\mathrm{FICl}$ score to assess the synergy/antagonism potential of tebipenem in combination with an LpxC inhibitor (Black), azithromycin (dark grey), ciprofloxacin (Mid grey), ceftriaxone (light grey), and mecillinam (white) against laboratory isolates of S. flexneri, S. Typhimurium, and E. coli. The scale of interaction is highlighted. B) Bar chart showing the average FICl score to assess the synergy/antagonism potential of tebipenem in combination with an LpxC inhibitor against a range of clinical isolates of enteric bacteria. C) Bar chart showing the average $\mathrm{FICl}$ score to assess the synergy/antagonism potential of tebipenem in combination with azithromycin against a range of clinical isolates of invasive and non-invasive Salmonella. 

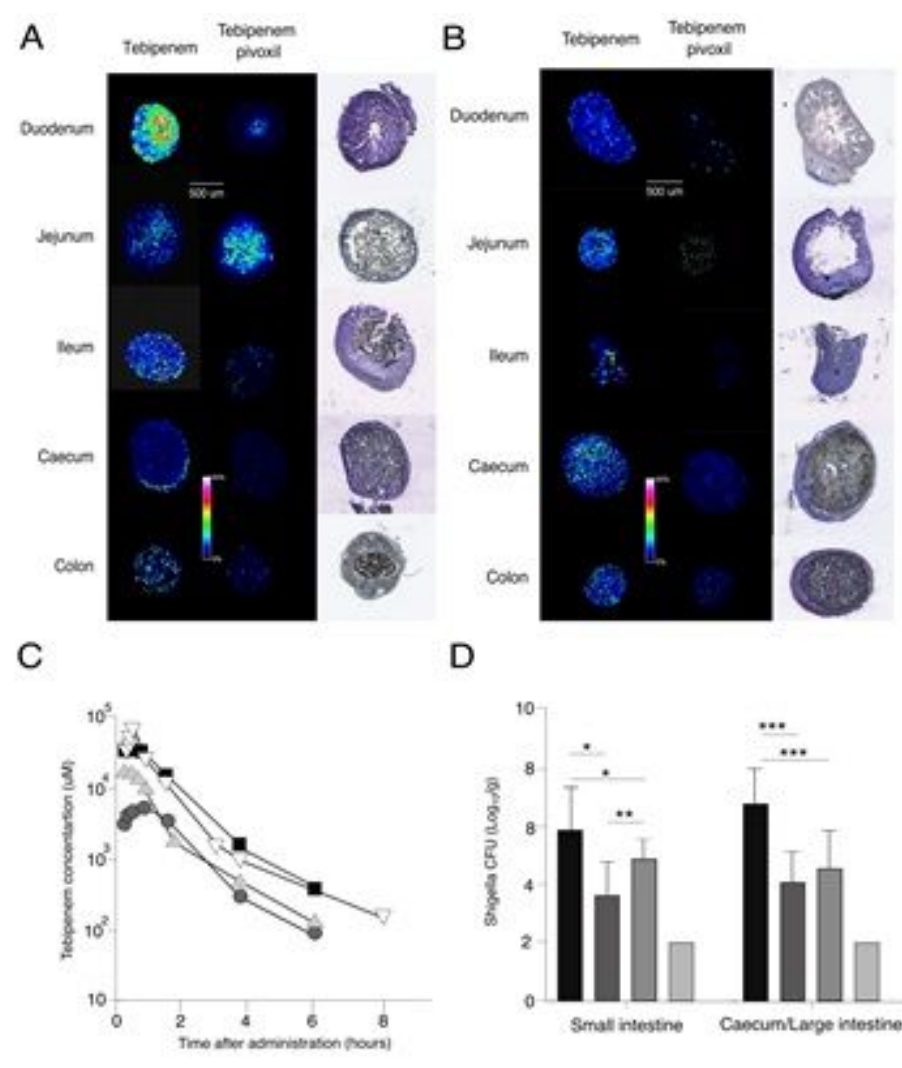

D

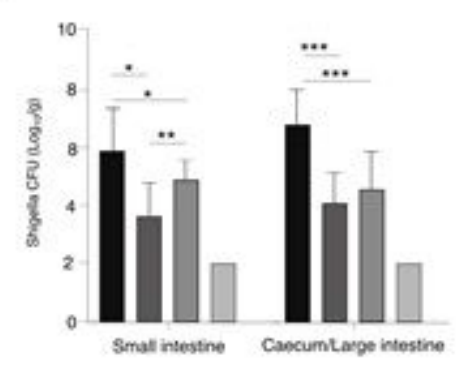

\section{Figure 4}

Pharmacodynamics and efficacy of tebipenem against Shigella in a murine infection model A) MALDITOF data showing the localisation and concentration of active tebipenem in different sections of the gastrointestinal tract (duodenum to colon) of the mouse after oral administration of tebipenem (left) and tebipenem pivoxil (middle) 15 minutes after administration. Drug intensity scale (0-60\%) and size scale are shown. A histology section of each compartment of the gastrointestinal tract is shown to highlight the localisation of active drug on the apical surface. B) MALDI-TOF data showing the localisation and concentration of active tebipenem in different sections of the gastrointestinal tract (as in A) 1 hour after administration. C) Pharmacokinetics of tebipenem in mice over 6 hours after the administration of $5 \mathrm{mg} / \mathrm{kg}$ intravenous tebipenem (grey triangles), $39 \mathrm{mg} / \mathrm{kg}$ subcutaneous tebipenem (white inverted triangles), $39 \mathrm{mg} / \mathrm{kg}$ oral tebipenem (grey circles), and $50 \mathrm{mg} / \mathrm{kg}$ oral tebipenem pivoxil (black squares). Notably, the drug concentration in all preparations remained above the estimated MIC of S. flexneri 2457T $(0.39 \mu \mathrm{M})$. D) The efficacy of $50 \mathrm{mg} / \mathrm{kg}$ oral tebipenem pivoxil (dark grey) and $39 \mathrm{mg} / \mathrm{kg}$ oral tebipenem (mid grey) in reducing the CFU/g of faeces, 24 hours after infection with a $1 \times 107$ intraperitoneal dose of S. flexneri 2457T compared to no drug (black) and $40 \mathrm{mg} / \mathrm{kg}$ ciprofloxacin. 

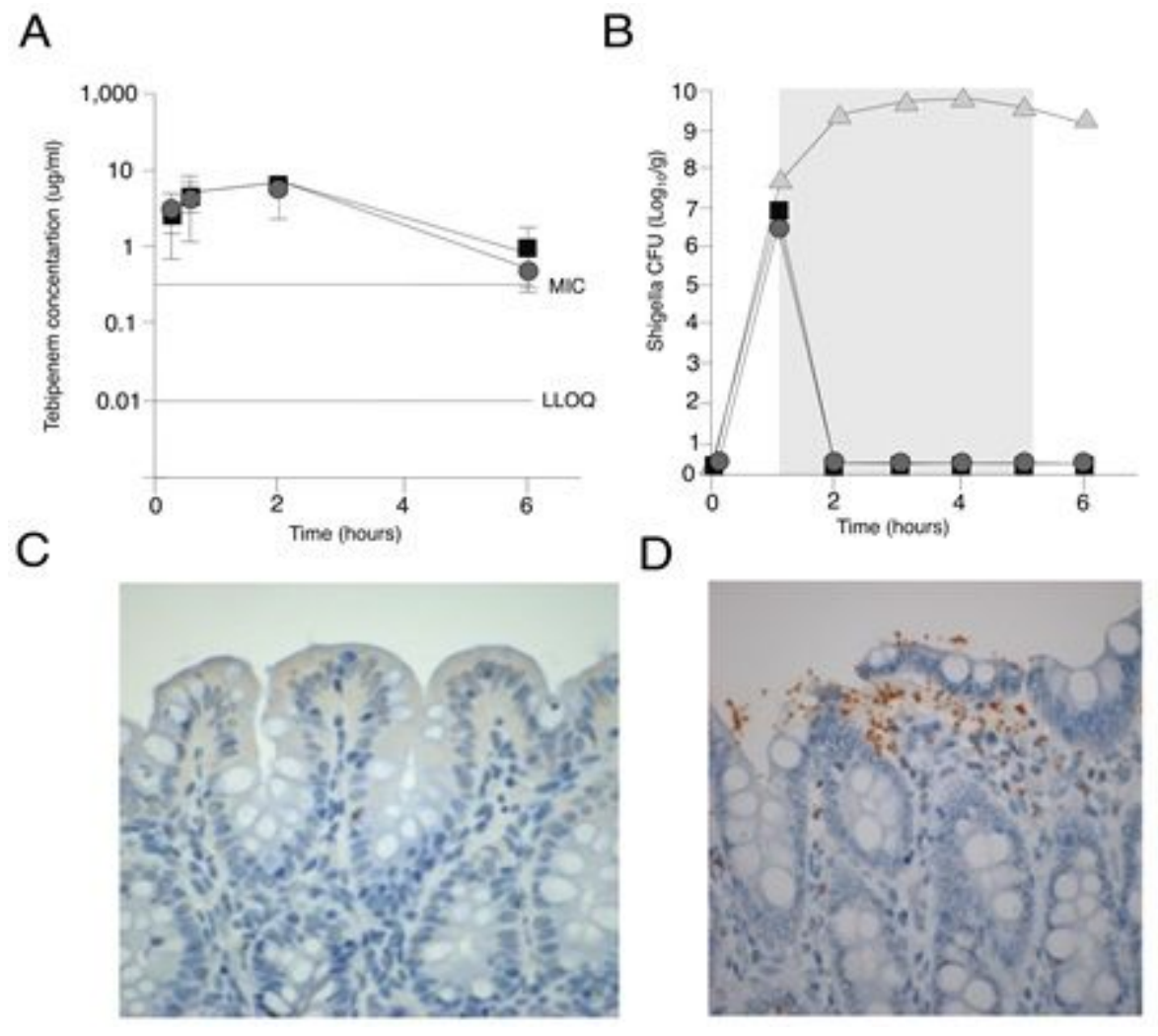

D

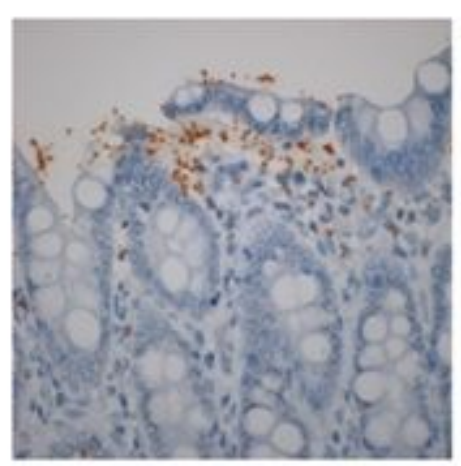

\section{Figure 5}

Pharmacodynamics and efficacy of tebipenem against Shigella in a gnotobiotic piglet infection model A) Pharmacodynamics of tebipenem in piglets over 6 hours after the administration of $50 \mathrm{mg} / \mathrm{kg}$ oral tebipenem pivoxil in Shigella infected (grey circles) and uninfected (black squares). The lower limit of quantitation (LLOQ) and the estimated MIC are shown. B) The efficacy of $50 \mathrm{mg} / \mathrm{kg}$ oral tebipenem pivoxil (grey circles) and oral $40 \mathrm{mg} / \mathrm{kg}$ ciprofloxacin (black squares) given daily for 5 days (grey shading) in reducing the $\mathrm{CFU} / \mathrm{g}$ of faeces (every 24 hours) after infection with a $1 \times 109$ oral dose of S. flexneri 2457T compared to no drug (black) over 6 days. C) Section of large intestine stained with $x$ taken on day 6 post infection in a gnotobiotic piglet treated with $50 \mathrm{mg} / \mathrm{kg}$ oral tebipenem pivoxil after infection with S. flexneri 2457T. D) Section of large intestine stained with $x$ taken on day 6 post infection in a gnotobiotic piglet after infection with S. flexneri $2457 T$

\section{Supplementary Files}

This is a list of supplementary files associated with this preprint. Click to download.

- SupplTable1AMRprofileforGSKuploadSep2018.xlsx

- Tables2.pdf 